\title{
Central extensions of the alternating group as Galois groups
}

\author{
by \\ Teresa Crespo (Barcelona)
}

1. Introduction. The aim of this paper is to study Galois embedding problems associated with central extensions of the alternating group with kernel a cyclic group. If $n \neq 6,7$, the nontrivial double cover $2 A_{n}$ of the alternating group $A_{n}$ is the universal central extension of $A_{n}$ and so, if

$$
1 \rightarrow C_{m} \rightarrow m A_{n} \rightarrow A_{n} \rightarrow 1
$$

is a nonsplit central extension of the alternating group $A_{n}$ with kernel a cyclic group $C_{m}$ of order $m$, we have a commutative diagram

$$
\begin{aligned}
& 1 \rightarrow C_{2} \rightarrow 2 A_{n} \rightarrow A_{n} \rightarrow 1 \\
& 1 \rightarrow \stackrel{\downarrow}{ } \begin{array}{l}
\downarrow \\
C_{m}
\end{array} \rightarrow m A_{n} \rightarrow A_{n} \rightarrow 1
\end{aligned}
$$

where the morphism $j$ is injective.

We identify $2 A_{n}$ with $j\left(2 A_{n}\right)$ and note that if $\left\{x_{s}\right\}$ is a system of representatives of $A_{n}$ in $2 A_{n}$, we can take it as a system of representatives of $A_{n}$ in $m A_{n}$ and so $m A_{n}$ is determined modulo isomorphisms. In the cases $n=6,7$, we denote by $m A_{n}$ the extension of $A_{n}$ fitting in the commutative diagram (1).

Let now $K$ be a field of characteristic different from $2, \bar{K}$ a separable closure of $K$, and $G_{K}$ the absolute Galois group of $K$. Let $f$ be an irreducible polynomial in $K[X]$, of degree $n \geq 4$ and with squared discriminant, $L$ a splitting field of $f$ contained in $\bar{K}$ and $G=\operatorname{Gal}(L \mid K)$. Let $E=K(x)$, for $x$ a root of $f$ in $L$. We consider $G$ as a subgroup of $A_{n}$ by means of its action on the set of $K$-embeddings of $E$ in $\bar{K}$. For $m G$ the preimage of $G$ in $m A_{n}$, we consider the embedding problem

$$
m G \rightarrow G \simeq \operatorname{Gal}(L \mid K) .
$$

Partially supported by grant PB89-0215 from CICYT. 
Now, if the embedding problem

$$
2 G \rightarrow G \simeq \operatorname{Gal}(L \mid K)
$$

is solvable, any embedding problem (2) is solvable. On the other hand, we know that the obstruction to the solvability of (3) is given by the Hasse-Witt invariant $\mathrm{w}\left(Q_{E}\right)$ of the quadratic form trace $Q_{E}=\operatorname{Tr}_{E \mid K}\left(X^{2}\right)$ ([5], Th. 1). Moreover, if it is solvable, the solutions can be computed effectively (cf. [1]).

If now $m=2^{r} l$, with $(l, 2)=1$, then $m A_{n}$ is the direct product of $C_{l}$ and $2^{r} A_{n}$. Therefore, if $L \mid K$ is a Galois extension with Galois group $G$, the general solution to the embedding problem $m G \rightarrow G \simeq \operatorname{Gal}(L \mid K)$ will be the composition $\widehat{L} . M$, for $\widehat{L}$ the general solution of the embedding problem $2^{r} G \rightarrow G \simeq \operatorname{Gal}(L \mid K)$ and $M \mid K$ running over the Galois extensions with Galois group $C_{l}$.

From now on, we assume then that $m=2^{r}$. That is, we consider embedding problems of the type

$$
2^{r} G \rightarrow G \simeq \operatorname{Gal}(L \mid K) .
$$

In the case $r=2$, we gave in [3] a criterion for the solvability of the embedding problem (4) and an effective way of computation for the solutions.

We note that if the embedding problem $2^{r} G \rightarrow G \simeq \operatorname{Gal}(L \mid K)$ is solvable, so is any embedding problem $2^{s} G \rightarrow G \simeq \operatorname{Gal}(L \mid K)$, with $s \geq r$. This comes from the fact that, for $r \geq 1$, if $c, d$ are generators of $C_{2^{r}}$ and $C_{2^{r+1}}$, respectively, $c^{i} x_{s} \rightarrow d^{2 i} x_{s}$ defines a morphism $2^{r} A_{n} \rightarrow 2^{r+1} A_{n}$ such that the diagram

is commutative.

$$
\begin{array}{ccc}
2^{r} A_{n} & \rightarrow & A_{n} \\
\downarrow & & \| \\
2^{r+1} A_{n} & \rightarrow & A_{n}
\end{array}
$$

On the other hand, the alternating groups $A_{4}$ and $A_{5}$ are subgroups of the projective linear group $\mathrm{PGL}(2, \mathbb{C})$ and the diagram

$$
\begin{aligned}
& 1 \rightarrow C_{2^{r}} \rightarrow 2^{r} A_{n} \quad \rightarrow \quad A_{n} \quad \rightarrow 1 \\
& \begin{array}{cccc}
\downarrow & \downarrow & \downarrow & (n=4,5)
\end{array} \\
& 1 \rightarrow \mathbb{C}^{*} \rightarrow \mathrm{GL}(2, \mathbb{C}) \rightarrow \operatorname{PGL}(2, \mathbb{C}) \rightarrow 1
\end{aligned}
$$

is commutative. The fact that the cohomology group $H^{2}\left(G_{K}, \mathbb{C}^{*}\right)$ is trivial, for $K$ a global or local field, gives that, for a given Galois realization $L \mid K$ of the group $A_{n}$, with $n=4,5$, the embedding problem (4) is solvable, for $r$ sufficiently big.

In the sequel, we give a criterion for the solvability of the embedding problems (4), and an explicit way of computing the solutions. We make a further study of the case $\mu_{2^{r-1}} \subset K$ in Section 3 and of the case $r=3$ in Section 4 . 
2. General case. The next proposition shows that solving the embedding problem (4) can be reduced to solving an embedding problem with kernel $C_{2}$.

Proposition 1. The embedding problem $2^{r} G \rightarrow G \simeq \operatorname{Gal}(L \mid K)$ is solvable if and only if there exists a Galois extension $K_{1} \mid K$ with Galois group $C_{2^{r-1}}$, such that $K_{1} \cap L=K$ and $\mathrm{w}\left(Q_{E}\right)=e^{*}(b)$ in $H^{2}\left(G_{K}, C_{2}\right)$, where $b \in H^{2}\left(C_{2^{r-1}}, C_{2}\right)$ is the element corresponding to the exact sequence $1 \rightarrow C_{2} \rightarrow C_{2^{r}} \rightarrow C_{2^{r-1}} \rightarrow 1$ and $e^{*}: H^{2}\left(C_{2^{r-1}}, C_{2}\right) \rightarrow H^{2}\left(G_{K}, C_{2}\right)$ the morphism induced by the epimorphism e $: G_{K} \rightarrow C_{2^{r-1}}$ corresponding to the extension $K_{1} \mid K$.

In this case, the set of solutions to the considered embedding problem is the union of the set of solutions to the embedding problems $2^{r} G \rightarrow G \times$ $C_{2^{r-1}} \simeq \operatorname{Gal}\left(L_{1} \mid K\right)$, where $L_{1}=L . K_{1}$ and $K_{1} \mid K$ runs over the extensions with the conditions given above.

Proof. Let $c$ be a generator of the group $C_{2^{r}}$. Let $\widehat{L}$ be a solution field to the considered embedding problem. For $L_{1}=\widehat{L}^{\left\langle c^{2^{r-1}}\right\rangle}$, we have $\operatorname{Gal}\left(L_{1} \mid K\right) \simeq 2^{r} G /\left\langle c^{2^{r-1}}\right\rangle \simeq G \times\left(C_{2^{r}} /\left\langle c^{2^{r-1}}\right\rangle\right)$. By taking $K_{1}=L_{1}^{G}$, we get $\operatorname{Gal}\left(K_{1} \mid K\right) \simeq C_{2^{r-1}}$ and $L \cap K_{1}=K$.

Now, $\widehat{L}$ is a solution to the embedding problem $2^{r} G \rightarrow G \times C_{2^{r-1}} \simeq$ $\operatorname{Gal}\left(L_{1} \mid K\right)$. For this embedding problem, the obstruction to the solvability is the product of the obstructions to the solvability of the embedding problems $2 G \rightarrow G \simeq \operatorname{Gal}(L \mid K)$ and $C_{2^{r}} \rightarrow C_{2^{r-1}} \simeq \operatorname{Gal}\left(K_{1} \mid K\right)$. For the first, as noted above, it is $\mathrm{w}\left(Q_{E}\right)$ and for the second $e^{*}(b)$.

Let us now assume that there exists a Galois extension $K_{1} \mid K$ with the conditions as in the proposition, and let $L_{1}=L . K_{1}$. We consider the embedding problem $2^{r} G \rightarrow G \times C_{2^{r-1}} \simeq \operatorname{Gal}\left(L_{1} \mid K\right)$. The obstruction to its solvability is $\mathrm{w}\left(Q_{E}\right) \otimes e^{*}(b)=1$ and, if $\widehat{L}$ is a solution, we have a commutative diagram

$$
\begin{array}{cccc}
\operatorname{Gal}(\widehat{L} \mid K) & \rightarrow & & \operatorname{Gal}(L \mid K) \times \operatorname{Gal}\left(K_{1} \mid K\right) \\
\simeq \downarrow & & & \simeq \\
2^{r} G & \rightarrow & G \times C_{2^{r-1}}
\end{array}
$$

and so, $\widehat{L}$ is a solution to the embedding problem $2^{r} G \rightarrow G \simeq \operatorname{Gal}(L \mid K)$.

Now, if the element $b$ is the second Stiefel-Whitney class of some orthogonal representation of the group $C_{2^{r-1}}$, the element $e^{*}(b)$ can be computed effectively by means of a formula of Fröhlich (cf. [4], Th. 3). Assuming that this is the case, we will give an explicit method of computation of the solutions.

We denote by $\varrho_{1}$ the orthogonal representation of the group $G$ obtained by embedding $A_{n}$ in the special orthogonal group $\mathrm{SO}\left(Q_{1}\right)$ of the standard 
quadratic form in $n$ variables. Let $\varrho_{2}: C_{2^{r-1}} \rightarrow O_{K}\left(Q_{2}\right)$ be a representation of $C_{2^{r-1}}$ in the orthogonal group $O_{K}\left(Q_{2}\right)$ of a quadratic form $Q_{2}$ over $K$ such that the second Stiefel-Whitney class sw $\left(\varrho_{2}\right)$ of $\varrho_{2}$ is equal to $b$. Taking into account [2], Prop. 3, we can assume that $\varrho_{2}$ is special and $\operatorname{sp} \circ \varrho_{2}=1$, where sp : $O_{K}\left(Q_{2}\right) \rightarrow K^{*} / K^{* 2}$ denotes the spinor norm. The obstruction to the solvability of the embedding problem $C_{2^{r}} \rightarrow C_{2^{r-1}} \simeq \operatorname{Gal}\left(K_{1} \mid K\right)$ is then given by $\mathrm{w}\left(Q_{2}\right) \otimes \mathrm{w}\left(Q_{2, \varrho_{2}}\right)$, where $Q_{2, \varrho_{2}}$ is the twisted form of $Q_{2}$ by $\varrho_{2}$. Moreover, for the orthogonal representation $\varrho:=\varrho_{1} \perp \varrho_{2}$, we have $\mathrm{sw}(\varrho)=\operatorname{sw}\left(\varrho_{1}\right) \otimes \operatorname{sw}\left(\varrho_{2}\right)([4],(1.7))$ and $Q_{\varrho}:=Q_{E} \perp Q_{2, \varrho_{2}}$ is the twisted form of $Q:=Q_{1} \perp Q_{2}$ by $\varrho$.

Let $C(Q)$ and $C\left(Q_{\varrho}\right)$ be the Clifford algebras of the quadratic forms $Q$ and $Q_{\varrho}$, let $C_{L_{1}}(Q)=C(Q) \otimes_{K} L_{1}$ and $C_{L_{1}}\left(Q_{\varrho}\right)=C\left(Q_{\varrho}\right) \otimes_{K} L_{1}$. For a Clifford algebra $C$, we denote by $C^{+}$the subalgebra of even elements and by $N$ the spinor norm. The fact that $Q_{\varrho}$ is the twisted form of $Q$ by $\varrho$ provides an isomorphism $f: C_{L_{1}}(Q) \rightarrow C_{L_{1}}\left(Q_{\varrho}\right)$ such that $f^{-1} f^{s}=\varrho(s)$ for all $s \in G \times C_{2^{r-1}}$. Let $n^{\prime}$ be the dimension of the orthogonal space of the form $Q$, and $e_{1}, e_{2}, \ldots, e_{n^{\prime}}$ an orthogonal basis. We are under the conditions of [2], Theorem 1 and so, we can state

THEOREM 1. If the embedding problem $2^{r} G \rightarrow G \times C_{2^{r-1}} \simeq \operatorname{Gal}\left(L_{1} \mid K\right)$ is solvable, there exists a $\mathbb{Z} / 2 \mathbb{Z}$-graduated algebra isomorphism $g: C(Q) \rightarrow$ $C\left(Q_{\varrho}\right)$ such that the element in $C_{L_{1}}^{+}\left(Q_{\varrho}\right)$ :

$$
z=\sum_{\varepsilon_{i}=0,1} v_{1}^{-\varepsilon_{1}} v_{2}^{-\varepsilon_{2}} \ldots v_{n^{\prime}}^{-\varepsilon_{n^{\prime}}} w_{n^{\prime}}^{\varepsilon_{n^{\prime}}} \ldots w_{2}^{\varepsilon_{2}} w_{1}^{\varepsilon_{1}},
$$

where $v_{i}=f\left(e_{i}\right), w_{i}=g\left(e_{i}\right), 1 \leq i \leq n^{\prime}$, is invertible.

The general solution to the considered embedding problem is then $\widetilde{L}=$ $L_{1}(\sqrt{r \gamma})$, where $\gamma$ is any nonzero coordinate of $N(z)$ in the basis $\left\{w_{1}^{\varepsilon_{1}} w_{2}^{\varepsilon_{2}} \ldots\right.$ $\left.\ldots w_{n^{\prime}}^{\varepsilon_{n^{\prime}}}\right\}, \varepsilon_{i}=0,1$, of $C_{L_{1}}\left(Q_{\varrho}\right)$, and $r$ runs over $K^{*} / K^{* 2}$.

3. Case $\mu_{2^{r-1}} \subset K$. We now assume that the field $K$ contains a root of unity $\zeta$ of precise order $2^{r-1}$. Under this hypothesis, we obtain

Proposition 2. The embedding problem $2^{r} G \rightarrow G \simeq \operatorname{Gal}(L \mid K)$ is solvable if and only if there exists an element $a$ in $K-L^{2}$ such that $\mathrm{w}\left(Q_{E}\right)=$ $(\zeta, a)$.

Proof. Let $K_{1}=K\left(\sqrt[2^{r-1}]{a}\right)$. We have $K_{1} \cap L=K$ and the obstruction to the solvability of the embedding problem $C_{2^{r}} \rightarrow C_{2^{r-1}} \simeq \operatorname{Gal}\left(K_{1} \mid K\right)$ is equal to the element $(\zeta, a) \in H^{2}\left(G_{K},\{ \pm 1\}\right)([4],(7.10))$. So we obtain the result by applying Proposition 1 .

We will now see how to compute the solutions to the embedding problem in this case. We assume $\mathrm{w}\left(Q_{E}\right)=(\zeta, a)$ for an element $a$ in $K$ and let 
$K_{1}=K(\alpha)$, where $\alpha=\sqrt[2 r-1]{a}, L_{1}=L . K_{1}$. Let $Q_{2}=\langle 2,-2,1,-\zeta, 1,-1\rangle$ and $\varrho_{2}$ be the orthogonal representation $C_{2^{r-1}} \rightarrow \mathrm{SO}\left(Q_{2}\right)$ given by

$$
\varrho_{2}(c)=\left(\begin{array}{cc}
R & 0 \\
0 & -I_{4}
\end{array}\right) \quad \text { where } \quad R=\left(\begin{array}{cc}
\frac{\zeta+\zeta^{-1}}{2} & \frac{\zeta-\zeta^{-1}}{2} \\
\frac{\zeta-\zeta^{-1}}{2} & \frac{\zeta+\zeta^{-1}}{2}
\end{array}\right)
$$

(cf. [2], Prop. 6).

Let $\varrho_{1}$ be the orthogonal representation $G \rightarrow A_{n} \rightarrow \mathrm{SO}_{n}(Q)$ and $\varrho=$ $\varrho_{1} \perp \varrho_{2}$. The twisted form of $Q$ by $\varrho$ is then $Q_{\varrho}=Q_{E} \perp\langle 2,-2, a,-\zeta a, a,-a\rangle$ and the solvability of the embedding problem $2^{r} G \rightarrow G \times C^{2^{r-1}} \simeq \operatorname{Gal}\left(L_{1} \mid K\right)$ implies $\mathrm{w}\left(Q_{\varrho}\right)=\mathrm{w}(Q)$. We can then apply the results obtained in [2]. Let $\left(x_{1}, \ldots, x_{n}\right)$ be a $K$-basis of $E$, and $\left\{s_{1}, \ldots, s_{n}\right\}$ the set of $K$-embeddings of $E$ in $\bar{K}$.

Let $M \in \mathrm{GL}\left(n+6, L_{1}\right)$ be the matrix

$$
M=\left(\begin{array}{cc}
M_{E} & 0 \\
0 & M_{a}
\end{array}\right)
$$

where

$$
M_{E}=\left(x_{j}^{s_{i}}\right)_{\substack{1 \leq i \leq n \\ 1 \leq j \leq n}}
$$

and

$$
M_{a}=\left(\begin{array}{cc}
N & 0 \\
0 & \sqrt{a} I_{4}
\end{array}\right) \quad \text { with } \quad N=\left(\begin{array}{cc}
\frac{\alpha+\alpha^{-1}}{2} & \frac{\alpha-\alpha^{-1}}{2} \\
\frac{\alpha-\alpha^{-1}}{2} & \frac{-\left(\alpha+\alpha^{-1}\right)}{2}
\end{array}\right) .
$$

We denote by $f: C_{L_{1}}(Q) \rightarrow C_{L_{1}}\left(Q_{e}\right)$ the isomorphism associated with the matrix $M^{-1}$. We have $f^{-1} f^{s}=\varrho(s), \forall s \in G \times C_{2^{r-1}}$.

We are then under the conditions of [2], Theorem 2 and can state

Theorem 2. If the forms $Q$ and $Q_{\varrho}$ are $K$-equivalent, we can choose $P \in \mathrm{GL}(n+6, K)$ such that

$$
P^{t}\left[Q_{\varrho}\right] P=[Q] \quad \text { and } \operatorname{det}(M P+I) \neq 0 .
$$

Then the general solution to the embedding problem $2^{r} G \rightarrow G \simeq \operatorname{Gal}(L \mid K)$ is

$$
\widetilde{L}=L_{1}(\sqrt{r \operatorname{det}(M P+I)}),
$$

with $r$ running over $K^{*} / K^{* 2}$.

P r o of. We consider the isomorphism of quadratic spaces associated with the matrix $P$ and take the isomorphism $g$ in Theorem 1 to be the extension of this isomorphism to the Clifford algebras. Then it is enough to compute the element $z$ as in [1], Theorem 4 . 
4. Extensions with kernel $C_{8}$. The next proposition gives the obstruction to the solvability of the considered embedding problem in the particular case $r=3$.

Proposition 3. The embedding problem $8 G \rightarrow G \simeq \operatorname{Gal}(L \mid K)$ is solvable if and only if there exist elements $a$ and $b$ in $K$ such that $b \notin K^{* 2}, b\left(a^{2}-4 b\right) \in$ $K^{* 2}$ and $\mathrm{w}\left(Q_{E}\right)=(-2, b) \otimes(-2 a,-1)$.

Proof. We note that an extension $K_{1} \mid K$ with Galois group $C_{4}$ is given by a polynomial $X^{4}+a X^{2}+b \in K[X]$, with $a$ and $b$ as in the proposition. By embedding $C_{4}$ in $S_{4}$ and using [4], Theorem 1, we conclude that the obstruction to the solvability of the embedding problem $C_{8} \rightarrow C_{4} \simeq \operatorname{Gal}\left(K_{1} \mid K\right)$ is equal to the element $(-2, b) \otimes(-2 a,-1) \in H^{2}\left(G_{K}, C_{2}\right)$.

We will now see how to compute the solutions to such an embedding problem. We assume that we are under the conditions of the proposition and let $K_{1} \mid K$ be the extension given by the polynomial $X^{4}+a X^{2}+b$, and $Q_{K_{1}}$ its quadratic trace form. We observe that $8 A_{n}$ is the pullback of the diagram

$$
\begin{aligned}
& A_{n} \times C_{4} \\
& \downarrow \\
& 2 A_{n+6} \quad \rightarrow \quad A_{n+6}
\end{aligned}
$$

where the vertical arrow is obtained by sending a generator of $C_{4}$ to the element (1234)(56) of $A_{6}$.

We then take $\varrho_{2}: C_{4} \rightarrow A_{6} \rightarrow \mathrm{SO}\left(Q_{2}\right)$, for $Q_{2}$ the standard quadratic form in 6 variables. We have $Q_{2, \varrho_{2}}=Q_{K_{1}} \perp\langle 2,2 b\rangle$ and so $Q_{\varrho}=Q_{E} \perp$ $Q_{K_{1}} \perp\langle 2,2 b\rangle$.

Now, we can apply the results obtained in [1]. We consider the matrix

$$
M=\left(\begin{array}{ccc}
M_{E} & 0 & 0 \\
0 & M_{a, b} & 0 \\
0 & 0 & M_{b}
\end{array}\right)
$$

where

$$
M_{E}=\left(x_{j}^{s_{i}}\right)_{\substack{1 \leq i \leq n \\
1 \leq j \leq n}}, \quad M_{a, b}=\left(y_{j}^{t_{i}}\right)_{\substack{1 \leq i \leq 4 \\
1 \leq j \leq 4}} \quad \text { and } \quad M_{b}=\left(\begin{array}{cc}
1 & \sqrt{b} \\
1 & -\sqrt{b}
\end{array}\right)
$$

for $\left(x_{j}\right)_{1 \leq j \leq n}$ a $K$-basis of $E,\left\{s_{i}\right\}_{1 \leq i \leq n}$ the set of $K$-embeddings of $E$ in $\bar{K},\left(y_{j}\right)_{1 \leq j \leq 4}$ a $K$-basis of $K_{1}$, and $\left\{t_{i}\right\}_{1 \leq i \leq 4}$ the set of $K$-embeddings of $K_{1}$ in $\bar{K}$.

We now assume $K=\mathbb{Q}$ and let $q=r_{2}(E)+r_{2}\left(K_{1}\right)+s g(b)$, where $r_{2}(E)$ (resp. $r_{2}\left(K_{1}\right)$ ) is the number of nonreal places of $E \mid \mathbb{Q}$ (resp. $K_{1} \mid \mathbb{Q}$ ) and $s g(b)=0$ (resp. 1) if $b>0$ (resp. $b<0$ ). We have that the signature of $Q_{\varrho}$ is $(n+6-q, q)$ and, by comparing $Q_{\varrho}$ with $Q_{q}:=-\left(X_{1}^{2}+\ldots+X_{q}^{2}\right)+$ $X_{q+1}^{2}+\ldots+X_{n+6}^{2}$, we see that the solvability of the embedding problem 
$8 G \rightarrow G \times C_{4} \simeq \operatorname{Gal}\left(L . K_{1} \mid \mathbb{Q}\right)$ implies $q \equiv 0(\bmod 4)$ and $Q_{\varrho} \mathbb{Q}$-equivalent to $Q_{q}$.

We now come back to the general hypothesis that $K$ is any field of characteristic different from 2 and, applying [1], Theorems 4 and 5, we obtain

Theorem 3. Assume $\mathrm{w}\left(Q_{E}\right)=(-2, b) \otimes(-2 a,-1)$ with $a, b \in K$ such that $b \notin K^{* 2}$ and $b\left(a^{2}-4 b\right) \in K^{* 2}$. Let $K_{1}$ be the splitting field of the polynomial $X^{4}+a X^{2}+b$ over $K$ and $L_{1}=L \cdot K_{1}$. Assume further that $Q_{\varrho}$ is $K$-equivalent to a quadratic form $Q_{q}:=-\left(X_{1}^{2}+\ldots+X_{q}^{2}\right)+X_{q+1}^{2}+\ldots+X_{n+6}^{2}$, with $q \equiv 0(\bmod 4)$. Let $P \in \operatorname{GL}(n+6, K)$ such that $P^{t}\left(Q_{\varrho}\right) P=\left(Q_{q}\right)$.

(a) If $q=0$, the solutions to the embedding problem $8 G \rightarrow G \times C_{4} \simeq$ $\operatorname{Gal}\left(L_{1} \mid K\right)$ are the fields $L_{1}(\sqrt{r \operatorname{det}(M P+I)})$, with $r$ running over $K^{*} / K^{* 2}$.

(b) If $q>0$, the solutions to the embedding problem $8 G \rightarrow G \times C_{4} \simeq$ $\operatorname{Gal}\left(L_{1} \mid K\right)$ are the fields $L_{1}(\sqrt{r \gamma})$, with $r$ running over $K^{*} / K^{* 2}$ and where the element $\gamma$ is given as a sum of minors of the matrix MP as in [1], Theorem 5 .

EXAMPLE. I thank J. Quer for the computation of this example. Let $f(X)=X^{4}-2 X^{3}+3 X^{2}+3 X+1$. The Galois group of $f$ over $\mathbb{Q}$ is the alternating group $A_{4}$ and we have $\mathrm{w}\left(Q_{E}\right)=-1$ in 5 and $\infty$ and $\mathrm{w}\left(Q_{E}\right)=1$ in all other primes. By applying [5], Theorem 1, we find that the embedding problem $2 A_{4} \rightarrow A_{4} \simeq \operatorname{Gal}(L \mid \mathbb{Q})$ is not solvable and, by noting that -1 is a square in $\mathbb{Q}_{5}$ and applying Proposition 2 , that the embedding problem $4 A_{4} \rightarrow A_{4} \simeq \operatorname{Gal}(L \mid \mathbb{Q})$ is also not solvable.

Now, we take $a=b=5$. We have $\mathrm{w}\left(Q_{E}\right)=(-2, b) \otimes(-2 a,-1)$ and the polynomial $X^{4}+5 X^{2}+5$ has Galois group $C_{4}$ over $\mathbb{Q}$. Then Proposition 3 gives that the embedding problem $8 A_{4} \rightarrow A_{4} \simeq \operatorname{Gal}(L \mid \mathbb{Q})$ is solvable.

In this case, the two fields $L$ and $K_{1}$ are totally imaginary and so we have $q=4$. By applying Theorem 3(b), we deduce that an element $\gamma$ in $L_{1}=L . K_{1}$ giving the solutions to the embedding problem $8 A_{4} \rightarrow A_{4} \times C_{4} \simeq \operatorname{Gal}\left(L_{1} \mid \mathbb{Q}\right)$ is

$$
\begin{aligned}
\gamma= & -262247420+283980105 x_{2}+29522845 x_{2}^{2} \\
& +x_{1}\left(211777885-179361840 x_{2}+116960680 x_{2}^{2}\right) \\
& +x_{1}^{2}\left(-23491885+35604590 x_{2}+12872070 x_{2}^{2}\right) \\
& +x_{1}^{3}\left(14803890+36883740 x_{2}+5569800 x_{2}^{2}\right) \\
& +r\left[-538192364-95254026 x_{2}-15821714 x_{2}^{2}\right. \\
& +x_{1}\left(-615191018+273851088 x_{2}-102712988 x_{2}^{2}\right) \\
& +x_{1}^{2}\left(504346598-192250828 x_{2}+21267108 x_{2}^{2}\right) \\
& \left.+x_{1}^{3}\left(-240082752+42410712 x_{2}-61752 x_{2}^{2}\right)\right] \\
& +r^{2}\left[-5967888+56866278 x_{2}+3865242 x_{2}^{2}\right.
\end{aligned}
$$




$$
\begin{aligned}
& +x_{1}\left(105764994-55214244 x_{2}+14643444 x_{2}^{2}\right) \\
& +x_{1}^{2}\left(-30093294+46771044 x_{2}-6310584 x_{2}^{2}\right) \\
& \left.+x_{1}^{3}\left(13943376-12532896 x_{2}+44136 x_{2}^{2}\right)\right] \\
& +r^{3}\left[-156513876-39659400 x_{2}-4293114 x_{2}^{2}\right. \\
& +x_{1}\left(-177964704+52921440 x_{2}-25362414 x_{2}^{2}\right) \\
& +x_{1}^{2}\left(155999832-27872568 x_{2}+1914552 x_{2}^{2}\right) \\
& \left.+x_{1}^{3}\left(-73067526+2548296 x_{2}-640404 x_{2}^{2}\right)\right]
\end{aligned}
$$

where $x_{1}$ and $x_{2}$ are two distinct roots of the polynomial $f$ and $r$ is a root of the polynomial $X^{4}+5 X^{2}+5$. We note that the extension $L_{1}(\sqrt{\gamma}) \mid \mathbb{Q}$ is nonramified outside 5 and 13 , which are the ramified primes in $L \mid K$.

\section{References}

[1] T. Crespo, Explicit construction of $\widetilde{A}_{n}$-type fields, J. Algebra 127 (1989), 452-461.

[2] - Explicit solutions to embedding problems associated to orthogonal Galois representations, J. Reine Angew. Math. 409 (1990), 180-189.

[3] - Extensions de $A_{n}$ par $C_{4}$ comme groupes de Galois, C. R. Acad. Sci. Paris 315 (1992), 625-628.

[4] A. Fröhlich, Orthogonal representations of Galois groups, Stiefel-Whitney classes and Hasse-Witt invariants, J. Reine Angew. Math. 360 (1985), 84-123.

[5] J.-P. Serre, L'invariant de Witt de la forme $\operatorname{Tr}\left(x^{2}\right)$, Comment. Math. Helv. 59 (1984), 651-676.

DEPARTAMENT D'ÀLGEBRA I GEOMETRIA

FACULTAT DE MATEMÀTIQUES

UNIVERSITAT DE BARCELONA

GRAN VIA DE LES CORTS CATALANES 585

08007 BARCELONA, SPAIN

E-mail: CRESPO@CERBER.UB.ES

Received on 20.4.1993

and in revised form on 10.11.1993 